\author{
(с) Л.В. Егшатян ${ }^{1,2}$, Н.Г. Мокрышева'
}

'ФГБУ Национальный медицинский исследовательский центр эндокринологии Минздрава России, Москва 2ФГБОУ ВО Московский государственный медико-стоматологический университет им. А.И. Евдокимова, Москва, Россия

Обоснование: на начальных стадиях хронической болезни почек (ХБП) основным патогенетическим звеном минерально-костных нарушений является снижение концентрации кальцитриола. Поддержание уровня витамина D и паратиреоидного гормона в пределах целевых значений снижает частоту развития сердечно-сосудистых, костных и почечных осложнений.

Цель: оценить эффективность нативных препаратов витамина D и селективного агониста рецепторов витамина D в коррекции вторичного гиперпаратиреоза (ВГПТ) у пациентов с ХБП 2-4 стадии в реальной клинической практике. Методы: обсервационное проспективное исследование для выявления эффективности и безопасности коррекции ВГПт у 54 пациентов с ХБП. Первый этап (16 недель) - компенсация субоптимальных уровней 25-гидроксикальциферола (25(ОН) D); при сохранении ВГПт - продление терапии до 24 недель. Второй этап (16 недель) - лечение колекальциферол-резистентного ВГПТ сочетанием колекальциферола с парикальцитолом. Всем пациентам определялись уровни 25(OH)D, общего кальция, альбумина, фосфора, креатинина крови, паратиреоидного гормона (ПТГ) и суточной экскреции кальция.

Результаты: через 8 недель терапии колекальциферолом в насыщающей дозе дефицит 25(OH)D был компенсирован, однако у 78\% пациентов сохранялся ВГПТ. Через 16 недель терапии ПТГ снизился значительно, однако достоверно только у пациентов с ХБП 2 (на 19,2\%, р <0,01) и 3 (на 31\%, p <0,05) стадии, в отличие от ХБП 4 стадии (на 17\%, p >0,05). ВГПТ сохранялся у 17 пациентов (31,5\%). Через 24 недель терапии нормализация ПтГ выявлена у всех пациентов с ХБП 2-й, у 15 (79\%) - 3-й и только у 9 (50\%) - 4-й стадии. Терапия колекальциферолом не оказала отрицательного влияния на уровень креатинина, кальция, фосфора сыворотки крови и суточной экскреции кальция.

Через 24 недель у пациентов с колекальциферол-резистентным ВГПТ ( $\mathrm{n=13)}$ начата комбинированная терапия колекальциферолом и парикальцитолом. Уровень ПтГ снизился с исходного $149.1 \pm 13.4$ пг/мл до $118.2 \pm 14.1$ пг/мл через 8 недель, и до $93.1 \pm 9.7$ пг/мл ( $<$ 0,05) через 16 недель терапии без эпизодов гиперкальциемии, гиперфосфатемии и гиперкальциурии. Компенсация ВГПТ достигнута у всех пациентов с 3-й и у 8 из 9 пациентов с 4-й стадией ХБП. Некомпенсированный пациент нуждался в увеличении дозы парикальцитола.

Заключение: данные литературы и собственный опыт указывают важность коррекции дефицита витамина D с целью профилактики или нормализации повышенных концентраций ПтГ на ранних стадиях, поскольку существуют ограничения эффективности нативных форм на поздних стадиях ХБП. Колекальциферол-резистентный ВГПТ хорошо компенсируется сочетанием колекальциферола и парикальцитола.

КЛЮЧЕВЫЕ СЛОВА: Околощитовидная железа; Вторичный гиперпаратиреоз; Колекальциферол; Парикальцитол; Активаторы рецепторов витамина D; Витамин D; Хроническая болезнь почек

\title{
THE EFFECTIVENESS OF NUTRITIONAL VITAMIN D SUPPLEMENTATION AND SELECTIVE VITAMIN D RECEPTOR AGONISTS TREATMENT ON SECONDARY HYPERPARATHYROIDISM IN CHRONIC KIDNEY DISEASES PATIENTS
}

\author{
(c) Lilit V. Egshatyan ${ }^{1,2}$, Natalia G. Mokrysheva'
}

${ }^{1}$ Endocrinology Research Centre, Moscow, Russia

${ }^{2}$ A.I. Evdokimov Moscow State University of Medicine and Dentistry, Moscow, Russia

Background: secondary hyperparathyroidism (SHPT) is an early complication of chronic kidney disease (CKD). Maintaining the level of 25(OH)D and parathyroid hormone concentrations in the target range reduce its associated complications (fractures and cardiovascular calcification).

Aims: to examine the effectiveness of vitamin D supplementation and selective vitamin D receptor agonists treatment on SHPT in CKD.

Material and methods: prospective observational study to evaluate the efficacy and safety of vitamin D therapy SHPT in 54 in patients with CKD. The first phase (24 weeks) - treatment of suboptimal 25-hydroxycalciferol (25(OH)D) levels. The second (16 weeks) - treatment colecalciferol-resistant SHPT by combination of cholecalciferol with paricalcitol. Blood samples were taken to assess parathyroid hormone (PTH), 25(OH)D, creatinine, calcium, phosphorus levels and calcium excretion. 
Results: After 8 weeks of cholecalciferol treatment all patients achieved 25(OH)D levels above $20 \mathrm{ng} / \mathrm{ml}$, however $78 \%$ of patients still had SHPT. After 16 weeks, the decrease of PTH was achieved in all patients, but significantly only in patients with CKD $2(19.2 \%, p<0.01)$ and $3(31 \%, p<0.05)$, compared with CKD 4 (17\%, p >0.05). After 24 weeks of therapy, PTH normalized in all patients with CKD 2, in 15 (79\%) with CKD 3 and in 9 (50\%) patients with CKD 4. Cholecalciferol treatment resulted in a substantial increase in $25(\mathrm{OH}) \mathrm{D}$ levels with minimal or no impact on calcium, phosphorus levels and kidney function.

After 24 weeks we initiated combination therapy (cholecalciferol and paricalcitol) for patients with colecalciferol-resistant SHPT $(n=13)$. PTH levels decreased from $149.1 \pm 13.4$ to $118.2 \pm 14.1 \mathrm{pg} / \mathrm{ml}$ at 8 weeks, and to $93.1 \pm 9.7 \mathrm{pg} / \mathrm{ml}(\mathrm{p}<0.05)$ at 16 weeks of treatment. No significant differences in serum calcium, phosphorus or urinary calcium levels. Normalization of PTH was achieved in all patients with CKD 3 and in 8 patients with stage 4. One patient with CKD 4 needed an increase in paricalcitol dose.

Conclusion: Cholecalciferol can be used in correcting vitamin D deficiency in patients with all stages of CKD, however, its effectiveness in reducing PTH in stage 4 is limited. Selective analogs, such as paricalcitol, were well-tolerated and effectively decreased PTH levels.

KEYWORDS: Parathyroid gland; secondary hyperparathyroidism; cholecalciferol; paricalcitol; vitamin D receptor activators; vitamin D; chronic kidney disease

\section{ОБОСНОВАНИЕ}

Распространенность недостаточности и дефицита витамина D в настоящее время можно считать пандемией - как среди детей, так и среди взрослого населения. Основной причиной дефицита Витамина D является недостаточная инсоляция. особенно у лиц молодого возраста, и значительно меньшее образование витамина D в коже у пожилых под воздействием ультрафиолетового излучения. Дефицит витамина D также развивается при недостаточном поступлении его с пищей. Выявлено, что в группу риска неадекватного потребления витамина D входит около половины земного населения [1]. Дополнительный вклад в распространенность субоптимальных уровней витамина D вносят различные препараты и заболевания, влияющие на его метаболизм. Дефицит витамина D широко распространен у пациентов с хронической болезнью почек (ХБП), и связан с развитием вторичного гиперпаратиреоза (ВГПТ) и неблагоприятными клиническими исходами. В связи с этим, рабочие группы и клинические рекомендации по минерально-костным нарушениям предлагают исследовать концентрации 25(OH)D у всех пациентов с ХБП, особенно начиная с 3-й стадии, и инициировать лечение при субоптимальных уровнях [2-7].
Сложность заключается в том, что не во всех клинических рекомендациях указывается пороговое значение 25(OH) $\mathrm{D}$ для начала лечения и его оптимальные целевые значения при наличии ХБП. Сравнительные характеристики международных рекомендаций, таких как K/DOQI (инициативы качества лечения заболеваний почек, 2003 г.) [5]; KDIGO (инициативы по улучшению глобальных исходов ХБП, 2009 и 2017г.) [6] и ERBP (рабочей группы европейских нефрологов, 2010 г.) [7], по определению и инициации лечения при субоптимальных уровнях 25(OH)D у пациентов с ХБП представлены в таблице 1.

Таким образом, на сегодняшний день руководство клинической практики KDIGO от 2017 года, рекомендует лечение дефицита и недостаточности витамина D абсолютно на всех стадиях ХБП, включая этап заместительной почечной терапии хроническим диализом, а также после проведения трансплантации почки, с использованием стратегий лечения, рекомендованных для населения в целом [6]. В предыдущих рекомендациях KDIGO от 2009 года, дефицит и недостаточность витамина $\mathrm{D}$ у реципиентов почки были определены как менее 40 нмоль/л (16 нг/мл) и 40-75 нмоль/л (16-30 нг/мл), соответственно [8], что в целом не отличается от градаций для населения в целом.

Таблица 1. Сравнение рекомендаций по определению и инициации лечения при недостаточности/дефиците 25(OH)D при хронической болезни почек

\section{KDOQI, 2003}

ХБП 3 и 4 стадий, если ПТГ выше целевого диапазона

Популяция пациентов для исследования 25(OH)D

Пороговое

значение

25(OH)D для вме-

шательства

При 25(OH)D менее 5 нг/мл пероральный прием эргокальциферола в дозе 50.000 МЕв неделю в течение

12 недель, затем 50.000 ME в месяц в течение 3 месяцев;

Методы коррекции 25(OH)D
При 25(OH)D 5-15 нг/мл прием 50.000 МЕ в неделю

в течение 4 недель, затем 50.000 МЕ в месяц в течение 5 месяцев;

При 25(ОН)D 16-30 нг/мл, прием 50.000 ME в месяц в течение 6 месяцев.

\section{ERBP, 2010} KDIGO, 2017 ХБП 3-5D и 1-5T* стадий

ХБП 3 и 4 стадий 12,5 нг/мл

нет

Рекомендуется использовать колекальциферол или другие аналоги 25(OH) D; конкретная стратегия лечения не указана.
Рекомендуются стратегии лечения, используемые в общей популяции; Никаких конкретных рекомендаций для использования колекальциферола или эргокальциферола нет.

*Примечание: ХБП - хроническая болезнь почек; 5D - диализная стадия ХБП; 1-5Т - посттрансплантационные стадии хронической болезни почки 
Таблица 2. Влияние Колекальциферола на уровни 25(ОН)D и паратиреоидного гормона у пациентов с хронической болезнью почек 2-4 стадий

\begin{tabular}{|c|c|c|c|c|c|c|c|c|}
\hline $\begin{array}{l}\text { Автор, } \\
\text { Год }\end{array}$ & $\begin{array}{c}\text { Тип иссле- } \\
\text { дования }\end{array}$ & $\begin{array}{c}\text { Количе- } \\
\text { ство паци- } \\
\text { ентов }\end{array}$ & $\begin{array}{c}\text { Колекальци- } \\
\text { ферол; кумуля- } \\
\text { тивная доза }\end{array}$ & $\begin{array}{l}\text { Дли- } \\
\text { тель- } \\
\text { ность }\end{array}$ & $\begin{array}{c}25(\mathrm{OH}) \\
D, \text { нг/мл } \\
\text { исход }\end{array}$ & $\begin{array}{c}\text { 25(OH) } \\
D, \text { нг/мл } \\
\text { конец }\end{array}$ & $\begin{array}{c}\text { ПТГ, } \\
\text { пг/мл } \\
\text { исход }\end{array}$ & $\begin{array}{l}\text { Динамика } \\
\text { ПТГ, пг/мл }\end{array}$ \\
\hline \multirow{2}{*}{$\begin{array}{l}\text { Dogan и } \\
\text { соавт., 2008г } \\
{[10]}\end{array}$} & \multirow[t]{2}{*}{ РКИ } & \multirow[t]{2}{*}{40 ХБП 3-4 } & $\begin{array}{c}\text { 300.000 МЕ одно- } \\
\text { кратно }\end{array}$ & 4 нед. & 8,5 & 17,8 & 368 & $\begin{array}{c}\text { Снижение } \\
(-89, \text { p<0,001) }\end{array}$ \\
\hline & & & Плацебо & & 6,8 & 7,2 & 273 & $\begin{array}{l}\text { Повышение } \\
(+15, p<0,004)\end{array}$ \\
\hline $\begin{array}{l}\text { Oksa и соавт., } \\
2008 г \text { [11] }\end{array}$ & РКИ & $\begin{array}{l}25 \text { ХБП 2; } \\
45 \text { ХБП 3; } \\
17 \text { ХБП } 4\end{array}$ & $\begin{array}{l}\text { 5.000 MЕ/нед. } \\
\text { (240.000 ME) }\end{array}$ & 48 нед. & 15 & 28 & 63 & $\begin{array}{c}\text { Снижение } \\
(-15, p<0,001)\end{array}$ \\
\hline $\begin{array}{l}\text { Alvarez и } \\
\text { соавт., 2012г } \\
{[12]}\end{array}$ & РКИ & 25 ХБП 3-4 & $\begin{array}{c}50.000 \mathrm{ME} / \text { нед. в } \\
\text { течение } 12 \text { нед., } \\
\text { далее } 50.000 \mathrm{ME} \\
\text { через неделю в } \\
\text { течение } 40 \text { нед. } \\
\text { (1.600.000 ME) }\end{array}$ & 52 нед. & 26,7 & 40,3 & 89 & $\begin{array}{c}\text { Снижение } \\
\text { к } 12 \text { нед. } \\
\text { (-19, p=0,01); } \\
\text { Нет достовер- } \\
\text { ного снижения } \\
\text { к } 52 \text { нед. }(-14)\end{array}$ \\
\hline $\begin{array}{l}\text { Cupisti и } \\
\text { соавт., 2015г } \\
\text { [13] }\end{array}$ & $\begin{array}{c}\text { Проспек- } \\
\text { тивное } \\
\text { когортное }\end{array}$ & 100 ХБП 2-4 & $\begin{array}{c}10.000 \text { ME/нед. } \\
\text { (480.000 ME) }\end{array}$ & 48 нед. & 12,3 & 22,4 & 135 & $\begin{array}{l}\text { Снижение } \\
(-17, p<0,05)\end{array}$ \\
\hline
\end{tabular}

Примечание: РКИ - рандомизированное контролируемое исследование, ХБП - хроническая болезнь почек

Таблица 3. Влияние Эргокальциферола на уровни 25(OH)D и паратиреоидного гормона у пациентов с хронической болезнью почек 3-5 стадий

\begin{tabular}{|c|c|c|c|c|c|c|c|c|}
\hline $\begin{array}{l}\text { Автор, } \\
\text { Год }\end{array}$ & $\begin{array}{l}\text { Тип ис- } \\
\text { следо- } \\
\text { вания }\end{array}$ & $\begin{array}{l}\text { Количе- } \\
\text { ство па- } \\
\text { циентов }\end{array}$ & $\begin{array}{c}\text { Эргокальцифе- } \\
\text { рол; } \\
\text { кумулятивная } \\
\text { доза }\end{array}$ & $\begin{array}{l}\text { Дли- } \\
\text { тель- } \\
\text { ность }\end{array}$ & $\begin{array}{c}25(\mathrm{OH}) \\
\mathrm{D}, \text { нг/мл } \\
\text { исход }\end{array}$ & $\begin{array}{c}\text { 25(OH) } \\
D, \text { нг/мл } \\
\text { конец }\end{array}$ & $\begin{array}{c}\text { ПтГ, } \\
\text { пг/мл } \\
\text { исход }\end{array}$ & $\begin{array}{l}\text { Динамика } \\
\text { ПТГ, пг/мл }\end{array}$ \\
\hline $\begin{array}{l}\text { Zisman и со- } \\
\text { авт., 2007г [14] }\end{array}$ & $\begin{array}{l}\text { Проспек- } \\
\text { тивное } \\
\text { когорт- } \\
\text { ное }\end{array}$ & $\begin{array}{l}24 \text { ХБП } 3 \\
28 \text { ХБП } 4\end{array}$ & $\begin{array}{c}\text { При 25(ОН) } \\
\text { D <15 нг/мл } \\
\text { 50.000 МЕ/нед.в те- } \\
\text { чение } 1 \text { мес.; да- } \\
\text { лее } 50.000 \text { МЕ/мес. } \\
\text { в течение } 3 \text { мес.; } \\
\text { далее } 1200 \text { ME/сут. } \\
\text { При 25(ОН)D } \\
\text { 15-25 нг/мл 50.000 } \\
\text { МЕ/нед. в тече- } \\
\text { ние } 1 \text { мес.; далее } \\
1200 \text { МЕ/сут. } \\
\text { При 25(ОН) } \\
\text { D >25 нг/мл } \\
\text { 1200-2000 МЕ/сут. }\end{array}$ & $\begin{array}{l}\text { 7,4 мес. } \\
\text { (ХБП 3) }\end{array}$ & $\begin{array}{c}20,3 \\
(\text { ХБП } 3)\end{array}$ & $\begin{array}{c}31,6 \\
(\text { ХБП 3) }\end{array}$ & 154 & $\begin{array}{l}\text { Снижение } \\
(-24, p=0,04)\end{array}$ \\
\hline $\begin{array}{l}\text { Gravesen } и \\
\text { соавт., 2013г } \\
{[15]}\end{array}$ & $\begin{array}{c}\text { Рандоми- } \\
\text { зирован- } \\
\text { ное }\end{array}$ & 43 ХБП 3-5 & 50.000 МЕ/нед & 6 нед. & 25,1 & 51,6 & 180 & $\begin{array}{c}\text { Нет достовер- } \\
\text { ного снижения } \\
(-12)\end{array}$ \\
\hline $\begin{array}{l}\text { Thimachai и } \\
\text { соавт.,2015г } \\
{[16]}\end{array}$ & РКИ & 68 ХБП 3-4 & $\begin{array}{c}\text { Двойная доза, } \\
\text { рекомендуемая } \\
\text { KDOQI, 2003г. }\end{array}$ & 8 нед. & 21 & 33,4 & 91 & $\begin{array}{c}\text { Снижение } \\
(-15, p=0,02)\end{array}$ \\
\hline $\begin{array}{l}\text { Susantitaphong } \\
\text { и соавт.,2017г } \\
{[17]}\end{array}$ & РКИ & 68 ХБП 3-4 & 40.000 МЕ/нед. & 12 нед. & 15,9 & 30,6 & 85 & $\begin{array}{l}\text { Нет достовер- } \\
\text { ного измене- } \\
\text { ния }(+1)\end{array}$ \\
\hline
\end{tabular}

Примечание: РКИ - рандомизированное контролируемое исследование, ХБП - хроническая болезнь почек

В клинических исследованиях показано, что как в общей популяции, так и у пациентов с ХБП 3-5 стадий (включая диализную) назначение лечебных доз колекальциферола не сопровождается развитием гиперкальциемии и другими побочными эффектами. Однако также показано, что назначение нативных форм витамина D не всегда, особенно у пациентов начиная с ХБП 3 стадии, по- ложительно влияет на компенсацию ВГПТ (таблицы 1-5). Следовательно, целесообразно ввести в рекомендации начало лечения неоптимальных уровней 25(OH)D уже на ранних стадиях ХБП, начиная с 1-й стадии.

В 2012 году опубликованы данные первого долгосрочного ( $\geq 1$ года), двойного слепого рандомизированного плацебо-контролируемого исследования терапии 
Таблица 4. Сравнительное влияние Коликальциферола/Эргокальциферола на уровни 25(ОН)D и паратиреоидного гормона у пациентов с хронической болезнью почек 3-5 стадий

\begin{tabular}{|c|c|c|c|c|c|c|c|c|}
\hline $\begin{array}{c}\text { Автор, } \\
\text { Год }\end{array}$ & $\begin{array}{l}\text { Тип ис- } \\
\text { следо- } \\
\text { вания }\end{array}$ & $\begin{array}{l}\text { Количе- } \\
\text { ство па- } \\
\text { циентов }\end{array}$ & $\begin{array}{c}\text { Колекальци- } \\
\text { ферол, Эрго- } \\
\text { кальциферол; } \\
\text { кумулятивная } \\
\text { доза }\end{array}$ & $\begin{array}{l}\text { Дли- } \\
\text { тель- } \\
\text { ность }\end{array}$ & $\begin{array}{c}25(\mathrm{OH}) \\
\mathrm{D}, \text { нг/мл } \\
\text { исход }\end{array}$ & $\begin{array}{c}\text { 25(ОН) } \\
\text { D, нг/мл } \\
\text { конец }\end{array}$ & $\begin{array}{c}\text { ПТГ, } \\
\text { пг/мл } \\
\text { исход }\end{array}$ & $\begin{array}{l}\text { Динамика } \\
\text { ПТГ, пг/мл }\end{array}$ \\
\hline $\begin{array}{l}\text { Wetmore и } \\
\text { соавт., 2016г } \\
{[18]}\end{array}$ & РКИ & 44 ХБП 3-5 & $\begin{array}{c}\text { Колекальцифе- } \\
\text { рол 50.000 МЕ/ } \\
\text { нед. (600.000 МЕ) } \\
\text { Эргокальциферол } \\
\text { 50.000 МЕ/нед. } \\
\text { (600.000 ME) }\end{array}$ & 12 нед. & 20,9 & 45,0 & 149 & $\begin{array}{c}\text { Снижение (-15, } \\
\text { р=0,02 между } \\
\text { группами) } \\
\text { Нет достовер- } \\
\text { ной динамики } \\
(+2)\end{array}$ \\
\hline
\end{tabular}

Примечание: РКИ - рандомизированное контролируемое исследование, ХБП - хроническая болезнь почек

Таблица 5. Влияние Колекальциферола на уровни 25(OH)D и паратиреоидного гормона у пациентов с хронической болезнью почек 5 диализной стадии

\begin{tabular}{|c|c|c|c|c|c|c|c|c|}
\hline $\begin{array}{l}\text { Автор, } \\
\text { Год }\end{array}$ & $\begin{array}{l}\text { Тип ис- } \\
\text { следо- } \\
\text { вания }\end{array}$ & $\begin{array}{l}\text { Количе- } \\
\text { ство па- } \\
\text { циентов }\end{array}$ & $\begin{array}{c}\text { Колекальцифе- } \\
\text { рол; кумулятив- } \\
\text { ная доза }\end{array}$ & $\begin{array}{l}\text { Дли- } \\
\text { тель- } \\
\text { ность }\end{array}$ & $\begin{array}{c}25(\mathrm{OH}) \\
\mathrm{D}, \text { нг/мл } \\
\text { исход }\end{array}$ & $\begin{array}{c}\text { 25(OH) } \\
\text { D, нг/мл } \\
\text { конец }\end{array}$ & $\begin{array}{c}\text { ПТГ, } \\
\text { пг/мл } \\
\text { исход }\end{array}$ & $\begin{array}{l}\text { Динамика } \\
\text { ПТГ, пг/мл }\end{array}$ \\
\hline $\begin{array}{l}\text { Tokmak и со- } \\
\text { авт., 2008г [19] }\end{array}$ & $\begin{array}{l}\text { Проспек- } \\
\text { тивное } \\
\text { когорт- } \\
\text { ное }\end{array}$ & 64 & $\begin{array}{c}20.000 \text { МЕ/нед. } \\
\text { (720.000 ME) }\end{array}$ & 36 нед. & 6,7 & 31,8 & 211 & $\begin{array}{c}\text { Нет достовер- } \\
\text { ной динамики } \\
(-5)\end{array}$ \\
\hline $\begin{array}{l}\text { Jean и соавт., } \\
\text { 2009г [20] }\end{array}$ & $\begin{array}{l}\text { Проспек- } \\
\text { тивное } \\
\text { когорт- } \\
\text { ное }\end{array}$ & 107 & $\begin{array}{c}100.000 \mathrm{ME} / \mathrm{Mec} . \\
\text { (1.500.000 ME) }\end{array}$ & 60 нед. & 12,8 & 42,4 & 294 & $\begin{array}{c}\text { Снижение } \\
(-104, p<0,05)\end{array}$ \\
\hline $\begin{array}{l}\text { Armas и соавт., } \\
\text { 2012г [21] }\end{array}$ & РКИ & 42 & $\begin{array}{c}\text { 10.333 МЕ/нед. } \\
\text { (154.995 ME) } \\
\text { Плацебо }\end{array}$ & 15 нед. & 13,3 & 36,9 & 174 & $\begin{array}{c}\text { Нет достовер- } \\
\text { ной динамики } \\
(-25,7) \\
\text { Нет достовер- } \\
\text { ной динамики } \\
(-8,3)\end{array}$ \\
\hline $\begin{array}{l}\text { Hewitt и соавт., } \\
\text { 2013г [22] }\end{array}$ & РКИ & 60 & $\begin{array}{c}10.333 \text { МЕ/нед. } \\
\text { (154.995 MЕ) } \\
\text { Плацебо }\end{array}$ & 24 нед. & $\begin{array}{l}18 \\
16\end{array}$ & $\begin{array}{l}35 \\
16\end{array}$ & $\begin{array}{l}335 \\
222\end{array}$ & $\begin{array}{l}\text { Нет достовер- } \\
\text { ной динамики } \\
\text { Нет достовер- } \\
\text { ной динамики }\end{array}$ \\
\hline $\begin{array}{l}\text { Dusilova- } \\
\text { Sulkova и } \\
\text { соавт., 2015г } \\
\text { [23] }\end{array}$ & $\begin{array}{l}\text { Проспек- } \\
\text { тивное } \\
\text { когорт- } \\
\text { ное }\end{array}$ & 34 & $\begin{array}{l}\text { 5.000 MЕ/нед. } \\
\text { (75.000 ME) }\end{array}$ & 15 & 7,4 & 27,5 & 205 & $\begin{array}{l}\text { Снижение } \\
(-34, p=0,05)\end{array}$ \\
\hline $\begin{array}{l}\text { Zitt и соавт., } \\
2015 г \text { [24] }\end{array}$ & $\begin{array}{l}\text { Проспек- } \\
\text { тивное } \\
\text { когорт- } \\
\text { ное }\end{array}$ & $\begin{array}{c}44 \text { ПГД } 12 \\
\text { ПД }\end{array}$ & $\begin{array}{c}100 \text { МЕ на кг веса } \\
\text { в нед. Сред- } \\
\text { няя недельная } \\
\text { доза } 7603 \mathrm{ME} \\
(200.000 \mathrm{ME})\end{array}$ & 26 нед & 9,9 & 26,1 & 362 & $\begin{array}{c}\text { Снижение } \\
(-65, p=0,001)\end{array}$ \\
\hline
\end{tabular}

Примечание: РКИ - рандомизированное контролируемое исследование, ХБП - хроническая болезнь почек, ПГД - программный гемодиализ, ПД перитонеальный диализ.

колекальциферолом в дозе 50.000 ME в неделю в течение 12 недель с последующим уменьшением дозы до 50.000 ME каждые две недели в течение 40 недель у пациентов с ранними стадиями (2 и 3) ХБП [9]. В ходе исследования выявлено, что пероральный прием колекальциферола в вышеуказанной дозе является безопасным и достаточным для поддержания нормальной концентрации 25(OH)D в сыворотке, а также для предотвращения недостаточности витамина D. Через год лечения выявлено достоверное снижение уровня ПТГ у пациентов с ВГПТ, по сравнению с плацебо. Вторая цель исследования за- ключалась в определении влияния колекальциферола на артериальное давление и уровень ФРФ-23, однако положительного влияния препарата на эти параметры найдено не было.

В литературе продемонстрирован положительный опыт применения колекальциферола (5000 ME в неделю) с селективным агонистом рецепторов витамина D (парикальцитолом) у пациентов с ВГПТ без повышения уровней кальция и фосфора в крови [31]. Синтезированный в 1985 г. парикальцитол, по сравнению с неселективными препаратами витамина D, хорошо снижает синтез ПТГ, в меньшей 
Таблица 6. Влияние Эргокальциферола на уровни 25(ОН)D и паратиреоидного гормона у пациентов с хронической болезнью почек 5 диализной стадии

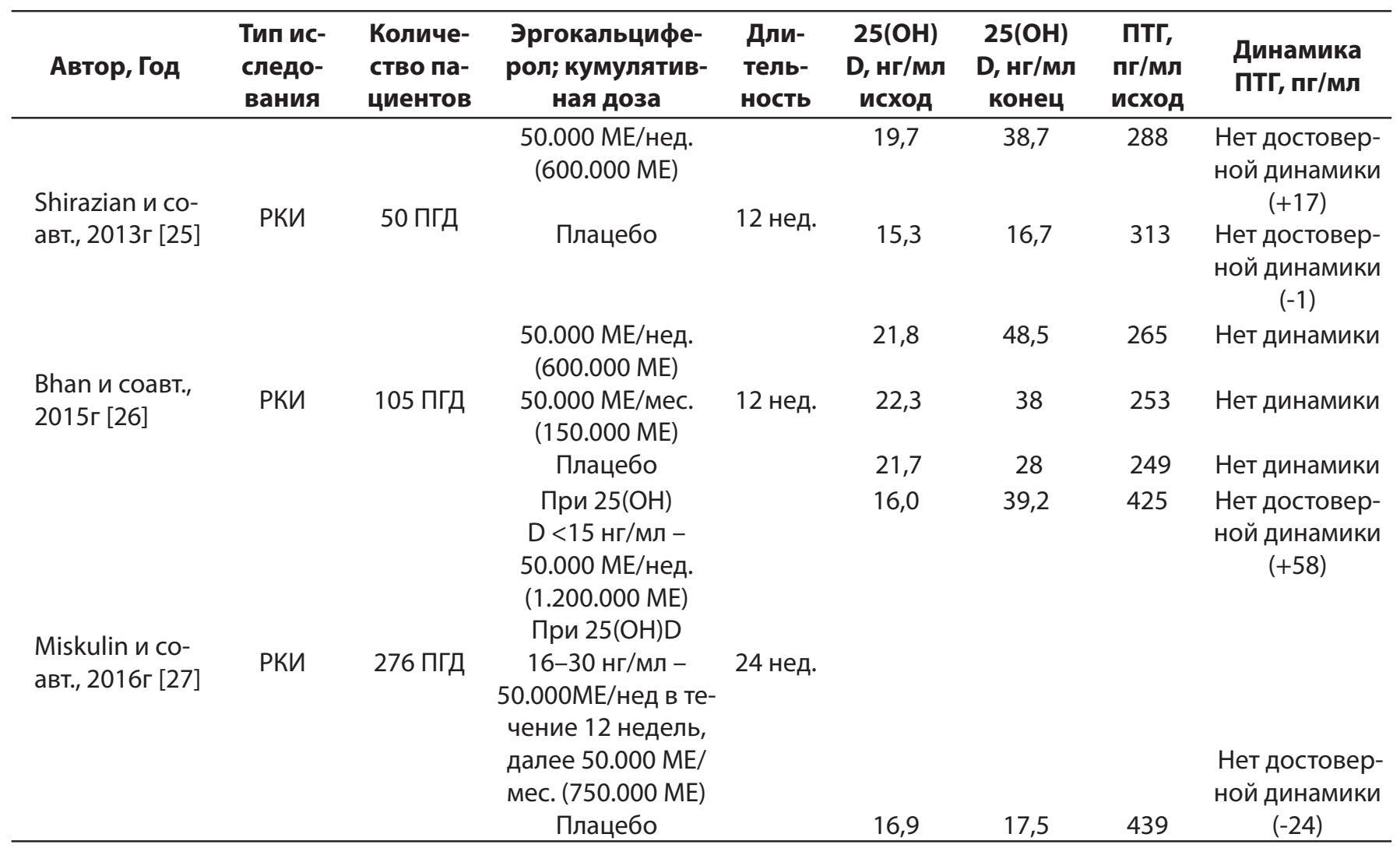

Примечание: РКИ - рандомизированное контролируемое исследование, ХБП - хроническая болезнь почек, Пгд - программный гемодиализ

степени влияя на абсорбцию кальция за счет сниженной афинности к рецепторам витамина D кишечника. В экспериментальной работе на крысах парикальцитол оказался в 10 раз менее активным, чем кальцитриол в отношении абсорбции кальция в кишечнике и мобилизации кальция из костной ткани [32]. Применение парикальцитола также не оказывает воздействия на экскрецию кальция с мочой, что очень важно для пациентов с ХБП [33]. Первоначально парикальцитол применялся только у диализных пациентов и внутривенно. Создание препарата в виде капсул для приема внутрь и. разрешение ее использования у пациентов, начиная с 3-й стадии ХБП, существенно расширило область его применения.

Согласно инструкции по применению парикальцитола, стартовая доза в капсулах определяется исходным уровнем ПТГ. При уровне ПТГ менее 500 пг/мл начальная доза препарата составляет 1 мкг 1 раз в день ежедневно или по 2 мкг 3 раза в неделю. Максимальная безопасная разовая доза в клинических исследованиях составила 32 мкг.

Несмотря на наличие данных о схожести профиля терапевтического действия препарата при разных режимах дозирования, в исследовании Н. Abboud и соавт. [34] выявлено, что при назначении парикальцитола пациентам 3-4 стадии ХБП наблюдается достоверно более выраженное снижение ПТГ при ежедневном приеме, по сравнению с приемом препарата 3 раза в неделю.

Необходимо отметить, что большинство исследований по ведению пациентов с минерально-костными нарушениями при ХБП были проведены в США и странах Западной Европы, и только ограниченные данные до- ступны за пределами этих стран. Мы начали собственное наблюдение чтобы продемонстрировать реальную клиническую практику ведения пациентов с ВГПТ при ХБП.

\section{ЦЕЛЬ}

Оценить эффективность и безопасность нативных препаратов витамина D и селективного агониста рецепторов витамина D в коррекции вторичного гиперпаратиреоза у пациентов с хронической болезнью почек 2-4-й стадии в реальной клинической практике.

\section{МЕТОДЫ}

\section{Дизайн исследования}

Обсервационное проспективное неконтролируемое исследование для выявления эффективности и безопасности коррекции ВГПТ у 54 пациентов с ХБП 2 - 4 стадии. Исследование состоит из:

- фазы скрининга,

- фазы коррекции субоптимальных уровней 25(OH)D и ВГПТ,

- фазы лечения колекальциферол-резистентного ВГПт. На первом этапе исследования перед началом лечения проводился скрининг пациентов, направленный на верификацию соответствия всем критериям включения/исключения, проведен сбор анамнеза и клинико-лабораторное обследование. Все пациенты, успешно прошедшие период скрининга, были разделены на 3 группы (ХБП 2, 3 и 4 стадии), согласно скорости клубочковой фильтрации. 
Таблица 7. Характеристики пациентов, включенных в исследование

\begin{tabular}{lccc}
\hline \multicolumn{1}{c}{ Параметр } & ХБП 2 ст. & ХБП 3 ст. & ХБП 4 ст. \\
\hline количество пациентов & 17 & 19 & 18 \\
возраст, лет & $50,3 \pm 10,5$ & $49,6 \pm 8,9$ & $56,7 \pm 9,6$ \\
25(ОН)D, нг/мл & $17,3 \pm 6,8$ & $15,8 \pm 5,9$ & $12,6 \pm 5,7$ \\
дефицит 25(ОН)D, n (\%) & $11(64,7)$ & $17(80,95)$ & $16(84,2)$ \\
ПТГ, пг/мл & $78,1 \pm 10,8$ & $99,6 \pm 19,3$ & $185,3 \pm 31,2$ \\
скоррСа, ммоль/л & $2,35 \pm 0,04$ & $2,23 \pm 0,09$ & $2,19 \pm 0,04$ \\
фосфор, ммоль/л & $1,1 \pm 0,08$ & $1,39 \pm 0,07$ \\
креатинин, мкмоль/л & $79,3 \pm 4,1$ & $1,25 \pm 0,05$ & $189,3 \pm 7,8$ \\
кальций суточной мочи, ммоль/сут & $4,7 \pm 0,08$ & $3,4 \pm 5,8$ & $2,39 \pm 0,07$ \\
\hline
\end{tabular}

Примечание: скоррСа - сывороточный общий кальций, скорректированный на уровень альбумина

Всем пациентам исходно, через 8, 16, 24 и 40 недель определялись уровни 25(OH)D, общего кальция, альбумина, фосфора, креатинина крови, паратиреоидного гормона (ПТГ) и суточной экскреции кальция.

\section{КРИТЕРИИ СООТВЕТСТВИЯ}

Критерии включения:

- ВГПТ на фоне ХБП 2-4-й стадии;

- Мужчины и женщины возраст от 30 до 70 лет.

Критерии невключения:

- Сердечная недостаточность III и IV ФК по NYHA;

- Психические заболевания, которые ограничивают адекватное сотрудничество;

- Онкологические заболевания, в том числе в анамнезе;

- Заболевания желудочно-кишечного тракта (печеночная недостаточность, мальабсорбция, хроническая диарея).

- Уровень фосфора более 2,0 ммоль/л и кальция скорректированного на альбумин менее 2,0 ммоль/л и более 2,55 ммоль/л в сыворотке крови (в двух анализах подряд);

- Уровень ПТГ ниже референсного диапазона для каждой стадии ХБП;

- Прием препаратов витамина D и кальция, фосфатснижающих препаратов, антиостеопоротических препаратов, препаратов влияющих на метаболизм витамина $D$, кальцимиметиков как минимум 6-ти месяцев до включения в исследование

Критерии исключения:

- Острый инфаркт миокарда;

- Острое нарушение мозгового кровообращения;

- Гиперфосфатемия на минимальных дозах препарата (уровень фосфора в сыворотке крови более 2,0 ммоль/л в двух анализах подряд)

- Гиперкальциемия на минимальных дозах препарата (в двух анализах подряд)

- Супрессия уровня паратиреоидного гормона на минимальных дозах препарата (в двух анализах подряд)

\section{Условия проведения}

В исследование были включены пациенты, проживающие в Москве и Московской области.

Продолжительность исследования

Фаза компенсации субоптимальных уровней 25(OH) D и коррекции ВГПТ - основной период наблюдения -
16 недель. При сохранении ВГПТ - продление приема колекальциферола до 24 недель. Через 24 недели, при наличии колекальциферол-резистентного ВГПТ - фаза лечения колекальциферол-резистентного ВГПТ комбинацией колекальциферола и селективного агониста рецепторов витамина D (парикальцитола), период наблюдения - 16 недель.

Описание медицинского вмешательства

С целью компенсации субоптимальных уровней 25(OH)D использовались стратегии лечения, рекомендованные для населения в целом, с применением насыщающих доз колекальциферола с дальнейшим переходом на поддерживающую терапию. Учитывая вышеописанные исследования, было решено продлить период назначения насыщающих доз с целью оценки безопасности и эффективности терапии. При субоптимальных уровнях рекомендуемая доза колекальциферола составила 50.000 ME в неделю: при дефиците 25(OH)D в течение 12 недель, при его недостаточности - в течение 8 недель, с дальнейшим переходом на 14.000 ME в неделю. Через 16 недель терапии при наличии резистентного ВГПТ на фоне компенсации субоптимальных уровней 25(OH)D, недельная доза колекальциферола была увеличена с 14.000 ME до 30.000 ME, период наблюдения 8 недель.

При отсутствии эффективности после 24 недель терапии колекальциферолом и формировании колекальциферол-резистентного ВГПТ рекомендован совместный прием колекальциферола в недельной дозе $7000 \mathrm{ME}$ и парикальцитола по 1 мкг 1 раз в день, ежедневно в течение 16 недель.

\section{Основной исход исследования}

Конечная точка исследования - нормализация показателей 25(OH)D и ПтГ без отрицательного влияния на уровень кальция, фосфора сыворотки крови и суточной экскреции кальция.

\section{Дополнительные исходы исследования}

Оценка креатинина и скорости клубочковой фильтрации на фоне терапии.

\section{Методы регистрации исходов}

Для регистрации основных и дополнительных исходов исследования использовались гормональный, биохимический анализ крови и мочи. 
Таблица 8. Характеристики пациентов, на фоне терапии колекальциферолом исходно и через 8 недель лечения

\begin{tabular}{|c|c|c|c|c|c|c|c|c|}
\hline \multicolumn{3}{|c|}{ ХБП 2 ст, } & \multicolumn{3}{|c|}{ ХБП 3 ст, } & \multicolumn{3}{|c|}{ ХБП 4 Ст, } \\
\hline \multicolumn{9}{|c|}{$\begin{array}{c}\text { 25(OH)D, нг/мл, } \\
\text { Исходно/через } 8 \text { недель }\end{array}$} \\
\hline $17,3 \pm 6,8$ & $28,3 \pm 5,7$ & $p<0,05$ & $15,8 \pm 5,9$ & $24,9 \pm 4,4$ & $p<0,05$ & $12,6 \pm 5,7$ & $24,1 \pm 4,2$ & $p<0,05$ \\
\hline \multicolumn{9}{|c|}{$\begin{array}{c}\text { Сывороточный общий кальций, скорректированный на уровень альбумина, ммоль/л } \\
\text { Исходно/через } 8 \text { недель }\end{array}$} \\
\hline $2,35 \pm 0,04$ & $2,33 \pm 0,07$ & $p>0,05$ & $2,23 \pm 0,09$ & $2,26 \pm 0,08$ & $p>0,05$ & $2,19 \pm 0,04$ & $2,21 \pm 0,02$ & $p>0,05$ \\
\hline \multicolumn{9}{|c|}{$\begin{array}{c}\text { Кальций в суточной моче, ммоль/сут } \\
\text { Исходно/через } 8 \text { недель }\end{array}$} \\
\hline $4,7 \pm 0,08$ & $4,84 \pm 0,09$ & $p>0,05$ & $3,4 \pm 0,05$ & $3,5 \pm 0,04$ & $p>0,05$ & $2,39 \pm 0,07$ & $2,42 \pm 0,06$ & $p>0,05$ \\
\hline \multicolumn{9}{|c|}{$\begin{array}{c}\text { Сывороточный фосфор, ммоль/л } \\
\text { Исходно/через } 8 \text { недель }\end{array}$} \\
\hline $1,1 \pm 0,08$ & $1,02 \pm 0,07$ & $p>0,05$ & $1,25 \pm 0,05$ & $1,29 \pm 0,06$ & $p>0,05$ & $1,39 \pm 0,07$ & $1,38 \pm 0,05$ & $p<0,05$ \\
\hline \multicolumn{9}{|c|}{$\begin{array}{l}\text { Креатинин крови, мкмоль/л } \\
\text { Исходно/через } 8 \text { недель }\end{array}$} \\
\hline $79,3 \pm 4,1$ & $80,1 \pm 3,2$ & $p>0,05$ & $121 \pm 5,8$ & $120,4 \pm 6,6$ & $p>0,05$ & $189,3 \pm 7,8$ & $188,2 \pm 8,9$ & $p>0,05$ \\
\hline \multicolumn{9}{|c|}{$\begin{array}{c}\text { ПТГ, пг/мл } \\
\text { Исходно/через } 8 \text { недель }\end{array}$} \\
\hline $78,1 \pm 18,3$ & $70,3 \pm 19,9$ & $p>0,05$ & $99,6 \pm 19,3$ & $84,3 \pm 17,1$ & $p>0,05$ & $185,3 \pm 31,2$ & $181 \pm 17,6$ & $p>0,05$ \\
\hline
\end{tabular}

Этическая экспертиза

Проведение научно-исследовательской работы одобрено ЛЭК ФГБУ «Эндокринологический научный центр». Выписка из протокола №2 от 10.02.16.

\section{Статистический анализ}

Статистическую обработку полученных данных проводили с помощью пакета прикладных программ STATISTICA (ВEPCИЯ 6). Числовые данные приведены в виде средних значений со стандартным отклонением $(\mathrm{M} \pm \mathrm{SD})$. Сравнение независимых выборок выполняли с помощью критерия Манна-Уитни. Статистически значимым считали значения критериев и коэффициентов, соответствующие $\mathrm{p}<0,05$.

\section{РЕЗУЛЬТАТЫ}

\section{Объекты (участники) исследования}

Пациенты, включенные в исследование, были разделены на три группы в зависимости от скорости клубочковой фильтрации (таблица 7). В группу ХБП 2 стадии вошли 17, ХБП 3 стадии - 19 и ХБП 4 стадии - 18 пациентов. Из таблицы 7 видно, что медиана концентрации 25(OH) D ниже референсного диапазона у всех пациентов. При этом у большинства (64,7\%; 80,95\% и 84,2\% с ХБП 2, 3 и 4 стадии, соответственно) диагностирован дефицит витамина D. Медиана сывороточного общего кальция, скорректированного на уровень альбумина и фосфора, а также суточной экскреции кальция были в пределах референсного диапазона во всех группах.

\section{Основные результаты исследования}

На фоне терапии колекальциферолом в насыщающей дозе в течение 8 недель наблюдалось успешное восполнение дефицита витамина D без отрицательного влияния терапии на уровни сывороточного кальция, фосфора и суточной экскреции кальция во всех исследуемых группах (таблица 8). Также не зафиксировано ухудшения функции почек на фоне проводимой терапии. Выявлено снижение уровня ПТГ, однако динамика была клинически недостоверной, и у большинства пациентов (78\%) сохранялся ВГПТ. После 8 недель лечения, терапия была продолжена по вышеуказанной схеме до суммарных 16 недель (таблица 9).

Через 16 недель терапии и наблюдения также не было зафиксировано побочных эффектов колекальциферола. Только в группе пациентов с ХБП 2 стадии отмечено достоверное повышение уровня кальциемии, однако, без эпизодов гиперкальциемии. К концу периода наблюдения дефицит 25(OH)D был компенсирован во всех группах. Уровень ПТГ снизился значительно во всех трех группах, однако достоверно только у пациентов с ХБП 2 и 3 стадии, что указывает на ограниченную эффективность нативных форм витамина D на предиализных стадиях ХБП.

Таким образом, после восполнения субоптимальных уровней 25(OH)D, через 16 недель терапии колекальциферолом, ВГПТ сохранялся у 17 пациентов (31,5\%):

- у 2 пациентов с ХБП 2 стадии (уровень 25(ОН)D 38 нг/мл и 41 нг/мл),

- у 7 пациентов с ХБП 3 стадии (уровень 25(ОН)D 39,5 \pm 7.8 нг/мл),

- у 10 пациентов с ХБП 4 стадии (уровень 25(ОН)D 37,9 \pm 8.1 нг/мл).

Согласно клиническим рекомендациям KDIGO от 2017 г., у пациентов с ХБП За-5 стадий желательно не использовать кальцитриол и аналоги витамина D peгулярно, а оставлять в качестве резерва для коррекции вторичного гиперпаратиреоза тяжелого и прогрессивного течения [6]. Поскольку неэффективность терапии в исследованиях в среднем оценивается через 6 месяцев лечения, и, учитывая клинические рекомендации руководства KIDIGO от 2017 года, у пациентов с некомпенсированным ВГПт была увеличена недельная доза колекальциферола с 14.000 до $30.000 \mathrm{ME}$ под контролем параметров фосфорно-кальциевого обмена через 8 недель. К концу периода наблюдения компенсация ВГПТ была достигнута у всех пациентов с ХБП 2 стадии, у тро- 
Таблица 9. Характеристики пациентов, на фоне терапии колекальциферолом исходно и через 8 недель лечения

\begin{tabular}{|c|c|c|c|c|c|c|c|c|}
\hline \multicolumn{3}{|c|}{ ХБП 2 ст. } & \multicolumn{3}{|c|}{ ХБП 3 ст. } & \multicolumn{3}{|c|}{ ХБП 4 ст. } \\
\hline \multicolumn{9}{|c|}{$\begin{array}{c}\text { 25(OH)D, нг/мл. } \\
\text { Исходно/через } 16 \text { недель }\end{array}$} \\
\hline $17.3 \pm 6.8$ & $43.7 \pm 10.2$ & $p<0,05$ & $15.8 \pm 5.9$ & $42.3 \pm 6.2$ & $p<0,05$ & $12.6 \pm 5.7$ & $34.3 \pm 7.5$ & $p<0,05$ \\
\hline \multicolumn{9}{|c|}{$\begin{array}{c}\text { Сывороточный общий кальций, скорректированный на уровень альбумина, ммоль/л } \\
\text { Исходно/через } 16 \text { недель }\end{array}$} \\
\hline $2.35 \pm 0.04$ & $2.42 \pm 0.06$ & $p<0,05$ & $2.23 \pm 0.09$ & $2.3 \pm 0.07$ & $p>0,05$ & $2.19 \pm 0.04$ & $2.2 \pm 0.04$ & $p>0,05$ \\
\hline \multicolumn{9}{|c|}{$\begin{array}{l}\text { Кальций в суточной моче, ммоль/сут } \\
\text { Исходно/через } 16 \text { недель }\end{array}$} \\
\hline $4.7 \pm 0.08$ & $5.03 \pm 0.08$ & $p>0,05$ & $3.4 \pm 0.05$ & $3.63 \pm 0.07$ & $p>0,05$ & $2.39 \pm 0.07$ & $2.6 \pm 0.08$ & $p>0,05$ \\
\hline \multicolumn{9}{|c|}{$\begin{array}{l}\text { Сывороточный фосфор, ммоль/л } \\
\text { Исходно/через } 16 \text { недель }\end{array}$} \\
\hline $1.1 \pm 0.08$ & $1.13 \pm 0.05$ & $p>0,05$ & $1.25 \pm 0.05$ & $1.28 \pm 0.09$ & $p>0,05$ & $1.39 \pm 0.07$ & $1.41 \pm 0.04$ & $p<0,05$ \\
\hline \multicolumn{9}{|c|}{$\begin{array}{l}\text { Креатинин крови, мкмоль/л } \\
\text { Исходно/через } 16 \text { недель }\end{array}$} \\
\hline $79.3 \pm 4.1$ & $78.2 \pm 4.2$ & $p>0,05$ & $121 \pm 5.8$ & $122.9 \pm 5.9$ & $p>0,05$ & $189.3 \pm 7.8$ & $190,7 \pm 9.1$ & $p>0,05$ \\
\hline \multicolumn{9}{|c|}{$\begin{array}{c}\text { ПТГ, пг/мл } \\
\text { Исходно/через } 16 \text { недель }\end{array}$} \\
\hline $78.1 \pm 18.3$ & $\begin{array}{l}\text { Снижение } \\
\text { на } 19,2 \%\end{array}$ & $p<0,01$ & $99.6 \pm 19.3$ & $\begin{array}{c}\text { Снижение } \\
\text { на } 31 \%\end{array}$ & $\mathrm{p}<0,05$ & $185.3 \pm 31.2$ & $\begin{array}{c}\text { Снижение } \\
\text { на } 17 \%\end{array}$ & $p>0,05$ \\
\hline
\end{tabular}

их пациентов с ХБП 3 стадии и только у одного пациента с ХБП 4 стадии, эпизодов гиперкальциемии, гиперкальциурии и гиперфосфатемии выявлено не было.

Таким образом, после 24 недель терапии колекальциферолом, нормализация уровня ПтГ была выявлена:

- у всех (100\%) пациентов с ХБП 2 стадии,

- у 15 (79\%) пациентов с ХБП 3 стадии,

- у 9 (50\%) пациентов с ХБП 4 стадии.

Так как имеются раннее опубликованные данные о положительном эффекте комбинации двух форм препаратов витамина D (колекальциферола и парикальцитола) для компенсации ВГПТ без риска ухудшения минерального обмена, в нашем исследовании у пациентов с колекальциферол-резистентным ВГПТ ( $\mathrm{n}=13$, из них 4 ХБП 3 стадии, 9 - ХБП 4 стадии) была начата комбинированная терапия колекальциферолом и парикальцитолом. На фоне лечения колекальциферолом в недельной дозе 7000 МЕ и парикальцитолом в суточной дозе 1 мкг В течение 16 недель достигнута компенсация ВГПТ. Как видно из рисунка 1, сочетание колекальциферола с парикальцитолом оказалось эффективным методом

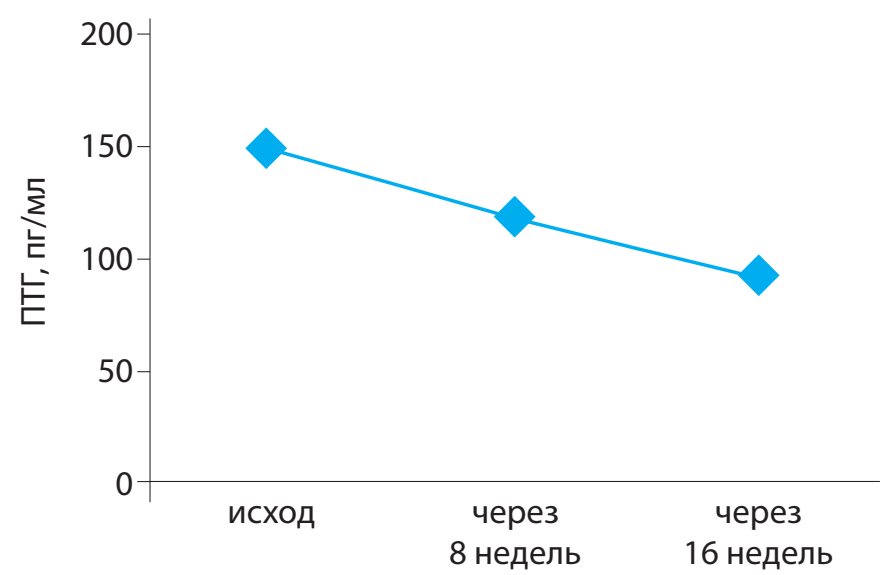

Рис. 1. Динамика уровня паратиреоидного гормона у пациентов с хронической болезнью почек 3-4-й стадии на фоне терапии колекальциферолом и парикальцитолом по отношению к снижению ПТГ при наличии ВГПТ, резистентного к терапии колекальциферолом. Выявлено

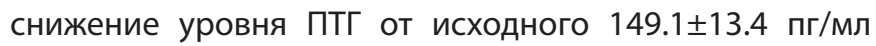
до $118.2 \pm 14.1$ пг/мл через 8 недель, и до $93.1 \pm 9.7$ пг/мл через 16 недель терапии ( $p<0,05)$. Компенсация ВГПТ достигнута у всех пациентов с ХБП 3 стадии и у 8 из 9 пациентов с ХБП 4 стадии. Некомпенсированный пациент нуждался в увеличении дозы парикальцитола.

Динамический контроль в течение 16 недель не выявил эпизодов гиперкальциемии, гиперфосфатемии и гиперкальциурии (рисунок 2). Все изменения показателей кальция, фосфора и креатинина были недостоверными ( $p>0,05)$ в течение всего периода наблюдения.

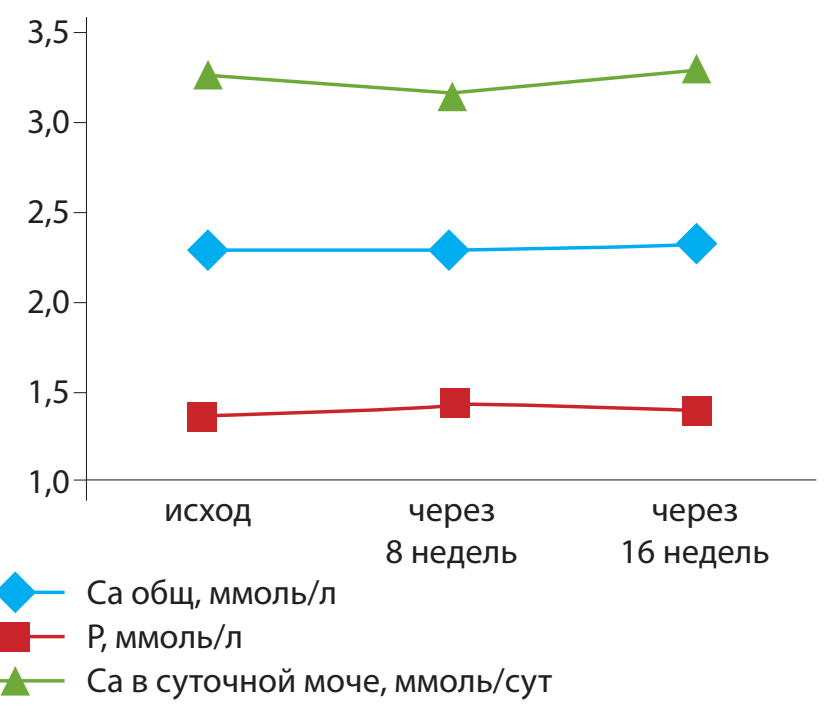

Рис. 2. Динамика показателей сывороточного кальция и фосфора, и суточной экскреции кальция у пациентов с хронической болезнью почек 3-4-й стадии на фоне терапии колекальциферолом и парикальцитолом

Примечание: Са - кальций, Са общ - сывороточный общий кальций, скорректированный на уровень альбумина, Р - сывороточный фосфор. 


\section{ОБСУЖДЕНИЕ}

Резюме основного результата исследования

На фоне компенсации субоптимальных уровней 25(OH)D и подключения к терапии парикальцитола при наличии резистентного к колекальциферолу ВГПТ достигнута основная цель исследования - нормализация уровня ПТГ без побочных эффектов терапии.

\section{Обсуждение основного результата исследования}

Исходя из данных литературы и собственного наблюдения, можно сделать вывод, что как в общей популяции, так и у пациентов с ХБП всех стадий (включая диализную) колекальциферол в насыщающих/лечебных дозах является безопасным и достаточным для компенсации дефицита и поддержания нормальной концентрации 25(OH)D в сыворотке крови. Также показано, что не всегда назначение нативных форм витамина D и восполнение субоптимальных уровней 25(OH)D, особенно у пациентов начиная с ХБП 3 стадии (особенно у пациентов с ХБП 4 и 5 стадий), положительно влияет на компенсацию ВГПТ [10-27]. В нашем исследовании стопроцентная компенсация ВГПТ на фоне нормальной концентрации 25(OH)D сыворотки крови была выявлена у пациентов с ХБП 2 стадии. Однако, несмотря на нормальные показатели 25(OH)D компенсация не достигнута у 4 (21\%) пациентов с ХБП 3 стадии и 9 (50\%) пациентов с ХБП 4 стадии. По данным литературы существует положительный опыт сочетания разных форм препаратов витамина D. Показано, что комбинированная терапия колекальциферолом (5000 ME в неделю) и селективным агонистом рецепторов витамина D (парикальцитолом) компенсирует ВГПТ у пациентов с ХБП без повышения уровней кальция и фосфора сыворотки крови [31]. Наши данные совпадают с литературными: из 13 пациентов 12 с колекальциферол-резистентным ВГПТ достигли целевого диапазона для ПТГ на фоне минимальных доз колекальциферола (7000 МЕ в неделю) и (парикальцитола 1 мкг в день) без риска развития гиперкальциемии, гиперкальциурии, гиперфосфатемии у ухудшения функции почек.

\section{Ограничения исследования}

Основным ограничением нашего исследования является маленький объем выборки и отсутствие групп контроля. Также ограничением можно считать отсутствие исследования таких важных параметров, как фактора роста фибробластов-23, белка klotho и так далее.

\section{Нежелательные явления}

В ходе исследования нежелательные явления, связанные с приемом холекальциферола и/или парикальцитола не зафиксированы.

\section{ЗАКЛЮЧЕНИЕ}

Поскольку дефицит витамина D играет важную роль в развитии ВГПТ у пациентов со сниженной функцией почек, поддержание нормального статуса витамина D является важным патогенетическим этапом профилактики и коррекции минерально-костных нарушений при ХБП. Учитывая высокую распространенность дефицита витамина D, целесообразно ввести в рекомендации мониторирование уровня 25(OH) D уже на ранних стадиях ХБП и при уровне 25(OH)D менее 30 нг/мл использовать препараты колекальциферола. Вышеприведенные исследования и наш собственный опыт показывают, что можно успешно восполнить дефицит витамина D и корректировать ВГПТ у пациентов на ранних стадиях ХБП, а на предиализных стадиях эффективность нативных форм витамина D ограничена. Имеющиеся данные свидетельствуют о том, что колекальциферол является безопасным препаратом у пациентов с ХБП. На предиализных стадиях ХБП восполнение дефицита витамина D не в состоянии компенсировать ВГПТ и повлиять на его прогрессию. В таких случаях препаратом выбора являются активные метаболиты и аналоги витамина D. При выборе предпочтение желательно отдавать препаратам, обладающим меньшим кальциемическим (при отсутствии выраженной гипокальциемии) и фосфатемическим эффектом и более выраженным эффектом ингибирования синтеза ПТГ - то есть селективным активаторам рецепторов витамина D (парикальцитолу). Сочетание колекальциферола с парикальцитолом хорошо контролирует ВГПТ у пациентов с резистентным к колекальциферолу ВГПТ без отрицательного влияния на показатели кальция, фосфора и почечную функцию.

Несмотря на все имеющиеся доказательства, все еще существует необходимость в проведении рандомизированных клинических исследований с целью определения целевого диапазона 25(OH)D у пациентов с ХБП, оценки эффективности комбинированной терапии прогормона и активных метаболитов витамина D.

\section{ДОПОЛНИТЕЛЬНАЯ ИНФОРМАЦИЯ}

Источники финансирования. Проведение исследования и подготовка статьи осуществлены на личные средства авторского коллектива.

Конфликт интересов. Авторы декларируют отсутствие явных и потенциальных конфликтов интересов, связанных с публикацией настоящей статьи.

Участие авторов. Все авторы внесли значимый вклад в проведение исследования и подготовку статьи, прочли и одобрили финальную версию статьи перед публикацией.

\section{СПИСОК ЛИТЕРАТУРЫ | REFERENCES}

1. Vitamin D Deficiency. N. Engl. J. Med. 2007;357(19):1980-1982. doi: 10.1056/NEJMc072359.

2. Ravani P, Malberti F, Tripepi G, et al. Vitamin D levels and patient outcome in chronic kidney disease. Kidney Int. 2009;75(1):88-95. doi: 10.1038/ki.2008.501

3. Wolf M, Shah A, Gutierrez O, et al. Vitamin D levels and early mortality among incident hemodialysis patients. Kidney Int. 2007;72(8):1004-1013. doi: 10.1038/sj.ki.5002451
4. Drechsler C, Pilz S, Obermayer-Pietsch B, et al. Vitamin $D$ deficiency is associated with sudden cardiac death, combined cardiovascular events, and mortality in haemodialysis patients. Eur. Heart J. 2010;31(18):2253-2261. doi: 10.1093/eurheartj/ehq246.

5. National Kidney F. K/DOQI clinical practice guidelines for bone metabolism and disease in chronic kidney disease. Am. J. Kidney Dis. 2003:42(4 Suppl 3):S1-201. 
6. Ketteler M, Block GA, Evenepoel P, et al. Executive summary of the 2017 KDIGO Chronic Kidney Disease-Mineral and Bone Disorder (CKD-MBD) Guideline Update: what's changed and why it matters. Kidney Int. 2017;92(1):26-36. doi: 10.1016/j.kint.2017.04.006

7. Goldsmith DJA, Covic A, Fouque D, et al. Endorsement of the Kidney Disease Improving Global Outcomes (KDIGO) Chronic Kidney Disease-Mineral and Bone Disorder (CKD-MBD) Guidelines: a European Renal Best Practice (ERBP) commentary statement. Nephrology Dialysis Transplantation. 2010;25(12):3823-3831. doi: 10.1093/ndt/gfa513.

8. Kidney Disease: Improving Global Outcomes CKDMBDWG. KDIGO clinical practice guideline for the diagnosis, evaluation, prevention, and treatment of Chronic Kidney Disease-Mineral and Bone Disorder (CKD-MBD). Kidney Int. Suppl. 2009(113):S1-130. doi: 10.1038/ki.2009.188

9. Alvarez JA, Law J, Coakley KE, et al. High-dose cholecalciferol reduces parathyroid hormone in patients with early chronic kidney disease: pilot, randomized, double-blind, placebo-controlled trial. The American Journal of Clinical Nutrition. 2012:96(3):672-679. doi: 10.3945/ ajcn.112.040642

10. Dogan E, Erkoc R, Sayarlioglu H, et al. Effect of Depot Oral Cholecalciferol Treatment on Secondary Hyperparathyroidism in Stage 3 and Stage 4 Chronic Kidney Diseases Patients. Ren. Fail. 2009;30(4):407410. doi: 10.1080/08860220801964210.

11. Oksa A, Spustova V, Krivosikova Z, et al. Effects of Long-Term Cholecalciferol Supplementation on Mineral Metabolism and Calciotropic Hormones in Chronic Kidney Disease. Kidney Blood Press. Res. 2008;31(5):322-329. doi: 10.1159/000157177.

12. Alvarez JA, Law J, Coakley KE, et al. High-dose cholecalciferol reduces parathyroid hormone in patients with early chronic kidney disease: a pilot, randomized, double-blind, placebo-controlled trial. The American Journal of Clinical Nutrition. 2012;96(3):672-679. doi: 10.3945/ajcn.112.040642

13. Cupisti A, Egidi MF, Vigo V, et al. Vitamin D status and cholecalciferol supplementation in chronic kidney disease patients: an Italian cohort report. Int. J. Nephrol. Renovasc. Dis. 2015:151. doi: 10.2147/ijnrd.s90968

14. Zisman AL, Hristova M, Ho LT, Spraque SM. Impact of Ergocalciferol Treatment of Vitamin D Deficiency on Serum Parathyroid Hormone Concentrations in Chronic Kidney Disease. Am. J. Nephrol. 2007;27(1):36-43. doi: 10.1159/000098561

15. Gravesen E, Hofman-Bang J, Lewin E, Olgaard K. Ergocalciferol treatment and aspects of mineral homeostasis in patients with chronic kidney disease stage 4-5. Scand. J. Clin. Lab. Invest. 2013;73(2):107116. doi: $10.3109 / 00365513.2012 .744464$

16. Thimachai P, Supasyndh O, Chaiprasert A, Satirapoj B. Efficacy of High vs. Conventional Ergocalciferol Dose for Increasing 25-Hydroxyvitamin D and Suppressing Parathyroid Hormone Levels in Stage III-IV CKD with Vitamin D Deficiency/Insufficiency: A Randomized Controlled Trial. J. Med. Assoc. Thai. 2015;98(7):643-648

17. Susantitaphong P, Nakwan S, Peerapornratana S, et al. A double-blind, randomized, placebo-controlled trial of combined calcitriol and ergocalciferol versus ergocalciferol alone in chronic kidney disease with proteinuria. BMC Nephrol. 2017;18(1). doi: 10.1186/s12882-017-0436-6.

18. Wetmore JB, Kimber C, Mahnken JD, Stubbs JR. Cholecalcifero v. ergocalciferol for 25 -hydroxyvitamin D $(25(\mathrm{OH}) \mathrm{D})$ repletion in chronic kidney disease: a randomised clinical trial. Br. J. Nutr. 2017:116(12):2074-2081. doi: 10.1017/s000711451600427x.
19. Tokmak F, Quack I, Schieren G, et al. High-dose cholecalciferol to correct vitamin D deficiency in haemodialysis patients. Nephrology Dialysis Transplantation. 2008;23(12):4016-4020. doi: 10.1093/ndt/gfn367.

20. Jean G, Souberbielle JC, Chazot C. Monthly cholecalciferol administration in haemodialysis patients: a simple and efficient strategy for vitamin D supplementation. Nephrology Dialysis Transplantation. 2009:24(12):3799-3805. doi: 10.1093/ndt/gfp370.

21. Armas LAG, Andukuri R, Barger-Lux J, et al. 25-Hydroxyvitamin D Response to Cholecalciferol Supplementation in Hemodialysis. Clin. J. Am. Soc. Nephrol. 2012;7(9):1428-1434. doi: 10.2215/cjn.12761211.

22. Hewitt NA, O'Connor AA, O'Shaughnessy DV, Elder GJ. Effects of Cholecalciferol on Functional, Biochemical, Vascular, and Quality of Life Outcomes in Hemodialysis Patients. Clin. J. Am. Soc. Nephrol. 2013;8(7):1143-1149. doi: 10.2215/cjn.02840312.

23. Dusilová-Sulková S, Šafránek R, Vávrová J, et al. Low-dose cholecalciferol supplementation and dual vitamin D therapy in haemodialysis patients. Int. Urol. Nephrol. 2014;47(1):169-176. doi: 10.1007/s11255-014-0842-7.

24. Zitt E, Sprenger-Mähr H, Mündle M, Lhotta K. Efficacy and safety of body weight-adapted oral cholecalciferol substitution in dialysis patients with vitamin D deficiency. BMC Nephrol. 2015;16(1). doi: 10.1186/s12882-015-0116-3.

25. Shirazian S, Schanler M, Shastry S, et al. The Effect of Ergocalciferol on Uremic Pruritus Severity: A Randomized Controlled Trial. J. Ren. Nutr. 2013;23(4):308-314. doi: 10.1053/j.jn.2012.12.007.

26. Bhan I, Dobens D, Tamez H, et al. Nutritional Vitamin D Supplementation in Dialysis: A Randomized Trial. Clin. J. Am. Soc. Nephrol. 2015;10(4):611-619. doi: 10.2215/cjn.06910714.

27. Miskulin DC, Majchrzak K, Tighiouart H, et al. Ergocalciferol Supplementation in Hemodialysis Patients With Vitamin D Deficiency: A Randomized Clinical Trial. J. Am. Soc. Nephrol. 2015;27(6):1801-1810. doi: 10.1681/asn.2015040468

28. Heaney RP, Recker RR, Grote J, et al. Vitamin D3Is More Potent Than Vitamin D2in Humans. J. Clin. Endocr. Metab. 2011;96(3):E447-E452. doi: 10.1210/jc.2010-2230.

29. Khazai NB, Judd SE, Jeng $L$, et al. Treatment and Prevention of Vitamin D Insufficiency in Cystic Fibrosis Patients: Comparative Efficacy of Ergocalciferol, Cholecalciferol, and UV Light. J. Clin. Endocr. Metab. 2009;94(6):2037-2043. doi: 10.1210/jc.2008-2012.

30. Trang HM, Cole DE, Rubin LA, et al. Evidence that vitamin D3 increases serum 25-hydroxyvitamin D more efficiently than does vitamin D2. The American Journal of Clinical Nutrition. 1998;68(4):854-858. doi: 10.1093/ajen/68.4.854

31. Zheng J-Q, Hou Y-C, Zheng C-M, et al. Cholecalciferol Additively Reduces Serum Parathyroid Hormone and Increases Vitamin D and Cathelicidin Levels in Paricalcitol-Treated Secondary Hyperparathyroid Hemodialysis Patients. Nutrients. 2016;8(11):708. doi: 10.3390/nu81 10708.

32. Brown AJ, Finch J, Slatopolsky E. Differential effects of 19-nor-1,25-dihydroxyvitamin D2 and 1,25-dihydroxyvitamin D3 on intestinal calcium and phosphate transport. J. Lab. Clin. Med. 2002;139(5):279284. doi: $10.1067 / \mathrm{mlc} .2002 .122819$

33. Coyne D, Acharya M, Qiu P, et al. Paricalcitol Capsule for the Treatment of Secondary Hyperparathyroidism in Stages 3 and 4 CKD. Am J. Kidney Dis. 2006:47(2):263-276. doi: 10.1053/j.ajkd.2005.10.007.

34. Abboud H, Coyne D, Smolenski O, et al. A Comparison of Dosing Regimens of Paricalcitol Capsule for the Treatment of Secondary Hyperparathyroidism in CKD Stages 3 and 4. Am. J. Nephrol. 2006:26(1):105-114. doi: 10.1159/000092033.

ИНФОРМАЦИЯ ОБ АВТОРАХ [AUTHORS INFO]

*Егшатян Лилит Ваниковна, к.м.н. [Lilit V. Egshatyan, MD, PhD]; адрес: Россия, 117036, Москва, улица Дм. Ульянова, д.11. [address: 11 Dm.Ulyanova street, 117036 Moscow, Russia]; адрес: Россия, 127473, Москва, ул. Делегатская 20, стр. 1 [address: $20 \backslash 1$ Delegatskaya ul., 127473, Moscow, Russia]; ORCID: http://orcid.org/0000-0001-8817-1901; eLibrary SPIN: 4552-5340; e-mail: lilit.egshatyan@yandex.ru.

Мокрышева Наталья Георгиевна, д.м.н. [Natalya G. Mokrisheva, MD, PhD]; ORCID: http://orcid.org/0000-0002-97179742; eLibrary SPIN: 5624-3875; e-mail: nm70@mail.ru 


\section{ЦИТИРОВАТЬ:}

Егшатян Л.В., Мокрышева Н.Г. Эффективность нативных препаратов витамина D и селективного агониста рецепторов витамина D в коррекции вторичного гиперпаратиреоза у пациентов с хронической болезнью почек. // Остеопороз и остеопатии. — 2018. - Т. 21. — №2. — C.12-22. doi: 10.14341/osteo9879

\section{TO CITE THIS ARTICLE:}

Egshatyan LV, Mokrisheva NG. The effectiveness of nutritional vitamin D supplementation and selective vitamin D receptor agonists treatment on secondary hyperparathyroidism in chronic kidney diseases patients. Osteoporosis and bone diseases. 2018;21(2):12-22. doi: 10.14341/osteo9879 